This is an Author's Accepted Manuscript of an article published as: CHRUSCIEL, P. and COSTA, J.L. (2009) Mass, angular-momentum and charge inequalities for axisymmetric initial data. Classical and Quantum Gravity. 26(23):235013 available online at: http://dx.doi.org/10.1088/0264-9381/26/23/235013 


\title{
Mass, angular-momentum, and charge inequalities for axisymmetric initial data
}

\author{
Piotr T. Chruściel \\ LMPT, Fédération Denis Poisson, Tours \\ Mathematical Institute and Hertford College, Oxford \\ João Lopes Costa \\ Lisbon University Institute - ISCTE \\ Mathematical Institute and Magdalen College, Oxford
}

September 30, 2009

\begin{abstract}
We present the key elements of the proof of an upper bound for angular-momentum and charge in terms of the mass for electro-vacuum asymptotically flat axisymmetric initial data sets with simply connected orbit space.
\end{abstract}

\section{Introduction}

In important recent work, Dain [6] has proved an upper bound for angularmomentum in terms of the mass for a class of maximal, vacuum, axisymmetric initial data sets. ${ }^{1}$ The aim of this work is to generalize Dain's inequality to include electric and magnetic charges. In fact, the heuristic argument behind Dain's original inequality generalizes to electro-vacuum as follows: the "standard picture of gravitational collapse" [7] is that the formation of

\footnotetext{
${ }^{1}$ The analysis of [6] has been extended in [3] to include all axisymmetric vacuum initial data, with simply connected orbit space, and manifolds which are asymptotically flat in the standard sense, allowing moreover several asymptotic ends.
} 
event horizons should occur generically in large families of space-times, with the exterior region approaching a Kerr-Newman metric asymptotically with time. Now, mass and Maxwell charges are conserved quantities, and the same is true for angular momentum if one further assumes axisymmetry, so the inequality (1.1) follows for all initial data for such a collapse. Besides its intrinsic interest, our inequality provides some support for this "standard picture", and in particular for weak cosmic censorship.

More precisely, consider a three dimensional electro-vacuum smooth initial data set $(M, g, K, E, B)$, where $M$ is the union of a compact set and of two asymptotically flat regions $M_{1}$ and $M_{2}$. Here $g$ is a Riemannian metric on $M, K$ is the extrinsic curvature tensor, $E$ is the electric field and $B$ the magnetic one, both divergence-free in electro-vacuum. We suppose that the initial data set is axisymmetric, by which we mean that it is invariant under an action of $\mathrm{U}(1)$, and maximal: $\operatorname{tr}_{g} K=0$. It is further assumed that $M / \mathrm{U}(1)$ is simply connected, so that the results of [2] can be used. The notion of asymptotic flatness is made precise in (2.1) and (2.3), where moreover $k \geq 6$ needs to be assumed when invoking [2]. We then have the following:

TheOREM 1.1 Under the conditions just described, let $m, \vec{J}, Q_{E}$ and $Q_{B}$ denote respectively the ADM mass, the ADM angular momentum, the total electric charge and the total magnetic charge of $M_{1}$ :

$$
Q_{E}=-\frac{1}{4 \pi} \int_{S_{\infty}} * F, \quad Q_{B}=\frac{1}{4 \pi} \int_{S_{\infty}} F
$$

Then

$$
m \geq \sqrt{\frac{|\vec{J}|^{2}}{m^{2}}+Q_{E}^{2}+Q_{B}^{2}} .
$$

A slightly more general version of Theorem 1.1 can be found in Theorem 2.1 below.

The reader should note an inequality relating area, angular momentum, and charge, proved for stationary Einstein-Maxwell black holes in [9], as well as the discussion of the Penrose inequality in electrovacuum of [11].

REMARK 1.2 We expect the equality to be attained only for the magnetically and electrically charged extreme Kerr-Newman space-times, which are unlikely to satisfy the hypotheses of Theorem 1.1. 
REMARK 1.3 If $M$ contains only one asymptotic flat end and $\partial M=\emptyset$ we have $\vec{J}=Q_{E}=Q_{B}=0$ (see (2.9) below), whence our interest in initial data sets containing two ends. One expects the result to generalize to several ends along the lines of [3], but a proof of this lies beyond the scope of this work.

REMARK 1.4 The proof applies to Einstein-Abelian Yang-Mills fields configurations, giving in this case

$$
m \geq \sqrt{\frac{|\vec{J}|^{2}}{m^{2}}+\left(\sum_{i} Q_{E_{i}}\right)^{2}+\left(\sum_{i} Q_{B_{i}}\right)^{2}},
$$

where the $Q_{E_{i}}$ 's and the $Q_{B_{i}}$ 's are the electric and magnetic charges associated with the $i$ 'th Maxwell field.

\section{Angular momentum and charge inequali- ties}

Recall that an asymptotically flat end is a region $M_{\text {ext }} \subset M$ diffeomorphic to $\mathbb{R}^{3} \backslash B(R)$, where $B(R)$ is a coordinate ball of radius $\mathrm{R}$, such that in local coordinates on $M_{\text {ext }}$ obtained from $\mathbb{R}^{3} \backslash B(R)$ we have, for some $k \geq 1,{ }^{2}$

$$
g_{i j}=\delta_{i j}+o_{k}\left(r^{-1 / 2}\right), \partial_{k} g_{i j} \in L^{2}\left(M_{\mathrm{ext}}\right), \quad K_{i j}=O_{k-1}\left(r^{-\lambda}\right), \lambda>\frac{5}{2} .
$$

(The asymptotic conditions on $g$ arise from the requirement of well defined ADM mass, with the integrability condition satisfied if, e.g., $\partial_{k} g_{i j}=$ $O\left(r^{-\alpha-1}\right)$, for some $\alpha>1 / 2$. The restriction on the decay rate of $K$ above arose already in the vacuum case, and can be traced back to the unnumbered equation after (2.37) in [3].)

The electric and magnetic fields $E$ and $B$ are defined to be the orthogonal projections to $T M$ of their space-time analogues

$$
E^{\mu}=F^{\mu}{ }_{\nu} n^{\nu}, \quad B^{\mu}=* F^{\mu}{ }_{\nu} n^{\nu},
$$

${ }^{2}$ We write $f=o_{k}\left(r^{-\alpha}\right)$ if the $\operatorname{limits}_{\lim } \lim _{r \rightarrow \infty} r^{\alpha+\ell} \partial_{k_{1}} \ldots \partial_{k_{\ell}} f$ vanish for all $0 \leq \ell \leq k$, and $f=O_{k}\left(r^{-\alpha}\right)$ if there exists a constant $C$ such that $\left|r^{\alpha+\ell} \partial_{k_{1}} \ldots \partial_{k_{\ell}} f\right| \leq C$ for all $0 \leq \ell \leq k$. 
where $F$ is the Maxwell two-form, and where $n$ is a unit normal to $M$, when embedded in a space-time. We assume that in the manifestly asymptotically flat coordinates we have

$$
E^{i}=O_{k-1}\left(r^{-\gamma-1}\right), \quad B^{i}=O_{k-1}\left(r^{-\gamma-1}\right), \gamma>3 / 4 .
$$

We will use the Einstein-Maxwell scalar constraint equation, which for maximal initial data reads

$$
{ }^{(3)} R=16 \pi \mu+|K|_{g}^{2}+2\left(|E|_{g}^{2}+|B|_{g}^{2}\right),
$$

where the function $\mu \geq 0$ represents the non-electromagnetic energy density and $|\cdot|_{g}$ denotes the norm of a vector with respect to the metric $g$.

To obtain our inequality we start by bounding (2.4) from below, as follows. By [2] there exists a coordinate system, with controlled asymptotic behaviour, in which the metric takes the form

$$
g=e^{-2 U+2 \alpha}\left(d \rho^{2}+d z^{2}\right)+\rho^{2} e^{-2 U}\left(d \varphi+\rho W_{\rho} d \rho+W_{z} d z\right)^{2} .
$$

Consider an orthonormal frame $e_{i}$ such that $e_{3}$ is proportional to the rotational Killing vector field

$$
\eta:=\partial_{\varphi}
$$

Let $\theta^{i}$ denote the dual co-frame; for definiteness we take

$$
\theta^{1}=e^{-U+\alpha} d \rho, \quad \theta^{2}=e^{-U+\alpha} d z, \quad \theta^{3}=\rho e^{-U}\left(d \varphi+\rho W_{\rho} d \rho+W_{z} d z\right) .
$$

We assume that the initial data are invariant under the flow of $\eta$; this implies the space-time equations $\mathscr{L}_{\eta} F=0$ and $\mathscr{L}_{\eta} * F=0$, where a star stands for the Hodge dual, and $\mathscr{L}$ denotes a Lie-derivative. A standard calculation, together with the hypothesis of simple-connectedness of $M / \mathrm{U}(1)$, implies then the existence of functions $\chi$ and $\psi$ such that

$$
\partial_{\alpha} \chi=F_{\mu \alpha} \eta^{\mu}, \quad \partial_{\alpha} \psi=* F_{\mu \alpha} \eta^{\mu}
$$

We then have

$$
\begin{aligned}
|E|_{g}^{2}+|B|_{g}^{2} & =\frac{e^{2 U}}{\rho^{2}}\left(\left(\partial_{n} \chi\right)^{2}+|D \chi|_{g}^{2}+\left(\partial_{n} \psi\right)^{2}+|D \psi|_{g}^{2}\right) \\
& \geq \frac{e^{2 U}}{\rho^{2}}\left(|D \chi|_{g}^{2}+|D \psi|_{g}^{2}\right) \\
& =\frac{e^{4 U-2 \alpha}}{\rho^{2}}\left(\left(\partial_{\rho} \chi\right)^{2}+\left(\partial_{z} \chi\right)^{2}+\left(\partial_{\rho} \psi\right)^{2}+\left(\partial_{z} \psi\right)^{2}\right)
\end{aligned}
$$


where $\partial_{n}$ denotes the derivative in the direction normal to the initial data hypersurface.

Writing

$$
4 \pi T_{\mu \nu} \eta^{\mu}=F_{\mu \alpha} F_{\nu}{ }^{\alpha} \eta^{\mu}-\frac{1}{4} F_{\alpha \beta} F^{\alpha \beta} \eta_{\nu}=\partial_{\alpha} \psi F_{\nu}^{\alpha}-\frac{1}{4} F_{\alpha \beta} F^{\alpha \beta} \eta_{\nu},
$$

we are now able to justify Remark 1.3, under the (reasonable) condition that

$$
F_{\mu \nu} \psi=o\left(r^{-2}\right) .
$$

First, the vanishing of the electric and magnetic charges is immediate. Next, the symmetry of the problem implies that $\vec{J}$ is aligned along the axis of rotation. Letting $J_{z}$ denote the component of angular-momentum along the rotation axis, by the Komar identity we obtain

$$
\begin{aligned}
16 \pi J_{z} & =\int_{S_{\infty}} \nabla^{\mu} \eta^{\nu} d S_{\mu \nu}=\frac{1}{2} \int_{M} \nabla_{\mu} \nabla^{\mu} \eta^{\nu} d S_{\nu} \\
& =\frac{1}{2} \int_{M} R_{\mu}{ }^{\nu} \eta^{\mu} d S_{\nu}=4 \pi \int_{M} T_{\mu}{ }^{\nu} \eta^{\mu} d S_{\nu} \\
& =-\int_{S_{\infty}} \psi \underbrace{\nabla_{\alpha} F^{\nu \alpha}}_{0} d S_{\nu}+\int_{M} \nabla_{\alpha}\left(\psi F^{\nu \alpha}\right) d S_{\nu}-\frac{1}{4} \int_{M} F_{\alpha \beta} F^{\alpha \beta} \eta^{\nu} d S_{\nu} \\
& =2 \int_{S_{\infty}} \psi F^{\nu \alpha} d S_{\nu \alpha}-\frac{1}{4} \int_{M} F_{\alpha \beta} F^{\alpha \beta} \underbrace{\eta^{\mu} n_{\mu}}_{0} d^{3} \mu_{g}=0
\end{aligned}
$$

where we have used the fact that $\eta$ is tangent to $M$, and where the first integral in the last line vanishes by (2.8).

As discussed in [6], in vacuum the one-form ${ }^{3}$

$$
\begin{aligned}
\lambda & :=2 \epsilon_{i j k} K^{j}{ }_{\ell} \eta^{k} \eta^{\ell} d x^{i} \\
& =2 \epsilon\left(\partial_{A}, \partial_{B}, \partial_{\varphi}\right) K\left(d x^{B}, \partial_{\varphi}\right) d x^{A} \\
& =2 g(\eta, \eta) \epsilon\left(e_{a}, e_{b}, e_{3}\right) K\left(\theta^{b}, e_{3}\right) \theta^{a}
\end{aligned}
$$

is closed. Here, as before, the upper case indices $A, B=1,2$ correspond to the coordinates $(\rho, z)$, while the lower case indices $a, b=1,2$ are frame indices. In electro-vacuum we have instead (see, e.g., [10])

$$
d(\lambda-2(\chi d \psi-\psi d \chi))=0,
$$

\footnotetext{
${ }^{3}$ We take this opportunity to point out a factor of 2 missing in the left-hand-side of Eq. (2.6) in [3], which affects numerical factors in some subsequent equations, but has no other consequences.
} 
and, since we have assumed that $M / \mathrm{U}(1)$ is simply connected, there exists a function $v$ such that

$$
\lambda=2(d v+\chi d \psi-\psi d \chi) .
$$

Then, writing $K_{b 3}$ for $K\left(e_{b}, e_{3}\right)$, and using $\epsilon_{a b}:=\epsilon\left(e_{a}, e_{b}, e_{3}\right) \in\{0, \pm 1\}$, we have

$$
2 \rho^{2} e^{-2 U}\left(K_{23} \theta^{1}-K_{13} \theta^{2}\right)=\lambda_{\rho} d \rho+\lambda_{z} d z
$$

equivalently

$$
K_{13}=-\frac{e^{3 U-\alpha}}{2 \rho^{2}} \lambda_{z}, \quad K_{23}=\frac{e^{3 U-\alpha}}{2 \rho^{2}} \lambda_{\rho}
$$

so that

$$
e^{2(\alpha-U)}|K|_{g}^{2} \geq 2 e^{2(\alpha-U)}\left(K_{13}^{2}+K_{23}^{2}\right)=\frac{e^{4 U}}{2 \rho^{4}}|\lambda|_{\delta}^{2}
$$

In [2] (compare $[1,6,8]$ ) it has been shown that

$$
\begin{aligned}
m= & \frac{1}{16 \pi} \int\left[{ }^{(3)} R+\frac{1}{2} \rho^{2} e^{-4 \alpha+2 U}\left(\rho W_{\rho, z}-W_{z, \rho}\right)^{2}\right] e^{2(\alpha-U)} d^{3} x \\
& +\frac{1}{8 \pi} \int(D U)^{2} d^{3} x .
\end{aligned}
$$

Inserting (2.7) and (2.14) into (2.15) we obtain

$$
\begin{aligned}
m & \geq \frac{1}{16 \pi} \int\left[{ }^{(3)} R e^{2(\alpha-U)}+2(D U)^{2}\right] d^{3} x \\
& \geq \frac{1}{8 \pi} \int\left[(D U)^{2}+\frac{e^{4 U}}{\rho^{4}}(D v+\chi D \psi-\psi D \chi)^{2}+\frac{e^{2 U}}{\rho^{2}}\left((D \chi)^{2}+(D \psi)^{2}\right)\right] d^{3} x,
\end{aligned}
$$

where, from now on, we use the symbol $D f$ to denote the gradient of a function $f$ with respect to the flat metric $\delta$, and where $(D f)^{2} \equiv|D f|_{\delta}^{2}$.

It follows from (2.6) that $\psi$ and $\chi$ are constant on each connected component $\mathscr{A}_{j}$ of the "axis"

$$
\mathscr{A}:=\{\rho=0\} \backslash\{z=0\} ;
$$

(2.12)-(2.13) then show that so is $v$. We set

$$
v_{j}:=\left.v\right|_{\mathscr{A}_{j}}, \quad \psi_{j}:=\left.\psi\right|_{\mathscr{A}_{j}}, \quad \chi_{j}:=\left.\chi\right|_{\mathscr{A}_{j}} .
$$

We have the following, from which Theorem 1.1 immediately follows: 
TheOREM 2.1 Let $(M, g, K, v, \chi, \psi)$ be a three dimensional smooth data set invariant under an action of $\mathrm{U}(1)$, where $M$ is the union of a compact set and of two asymptotically flat regions $M_{1}$ and $M_{2}$, in the sense of (2.1) together with (2.3), and where $v, \psi$ and $\chi$ are global potentials as in (2.12)-(2.13) and (2.6). Assume that (2.4) holds with $\mu \geq 0$. Let $m$ and $\vec{J}$ denote the ADM mass and angular momentum of $M_{1}$, and let $Q_{E}$ and $Q_{B}$ be the global electric and magnetic charges of $M_{1}$. If $M / \mathrm{U}(1)$ is simply connected, then

$$
m \geq \sqrt{\frac{|\vec{J}|^{2}}{m^{2}}+Q_{E}^{2}+Q_{B}^{2}} .
$$

The proof of Theorem 2.1 proceeds as follows. If the mass is infinite there is nothing to prove; under the current hypotheses this will be the case iff ${ }^{(3)} R \notin L^{1}(M)$. Otherwise, in view of (2.16), one considers the action

$$
I:=\int\left[(D U)^{2}+\frac{e^{4 U}}{\rho^{4}}(D v+\chi D \psi-\psi D \chi)^{2}+\frac{e^{2 U}}{\rho^{2}}\left((D \chi)^{2}+(D \psi)^{2}\right)\right] d^{3} x .
$$

One wishes to show that $I$ is bounded from below by the right-hand-side of our bound, which is the value of the corresponding action for the extreme Kerr-Newman solution with the same global Poincaré and Maxwell charges. The proof can be obtained by following the argument in [3], except for supplementary difficulties in several places related to the new structure of the term $\frac{e^{4 U}}{\rho^{4}}(D v+\chi D \psi-\psi D \chi)^{2}$. For example, when a cut-off function $\varphi_{\eta}$ is used in [3], the estimates do not appear to go through using the arguments there. This can be circumvented as follows: Let the quantities decorated with tildes refer to the extreme Kerr-Newman solution. Let $\theta=U, v, \chi, \psi$ and write

$$
\theta_{\eta}:=\varphi_{\eta} \theta+\left(1-\varphi_{\eta}\right) \tilde{\theta}=\varphi_{\eta}(\theta-\tilde{\theta})+\tilde{\theta} .
$$

Set

$$
\lambda_{\eta}:=D v_{\eta}+\chi_{\eta} D \psi_{\eta}-\psi_{\eta} D \chi_{\eta} .
$$

While a direct estimate of $\lambda_{\eta}$ in the most singular integrals in [3] does not appear to be immediate, it turns out that each summand in the identity

$$
\begin{aligned}
\lambda_{\eta}= & \varphi_{\eta} \lambda+\left(1-\varphi_{\eta}\right) \tilde{\lambda}+D \varphi_{\eta}(v-\tilde{v})+D \varphi_{\eta}(\tilde{\chi} \psi-\tilde{\psi} \chi) \\
& +\varphi_{\eta}\left(1-\varphi_{\eta}\right)\{(\psi-\tilde{\psi}) D(\chi-\tilde{\chi})-(\chi-\tilde{\chi}) D(\psi-\tilde{\psi})\}
\end{aligned}
$$


can be handled in a way similar to the original integrals in [3]. For example, one of the steps of the proof is to establish that

$$
\int_{\eta / 2<r<\eta} \frac{e^{4 U_{\eta}}}{\rho^{4}}\left|\lambda_{\eta}\right|^{2} d^{3} x \rightarrow_{\eta \rightarrow 0} 0,
$$

where $r^{2}=\sqrt{\rho^{2}+z^{2}}$. Recall that near $r=0$ the coordinates $(\rho, z)$ can be obtained from the usual cylindrical coordinates in the other asymptotically flat region, which we denote by $(\hat{\rho}, \hat{z})$, by an inversion $(\hat{\rho}, \hat{z})=\left(\frac{\rho}{r^{2}}, \frac{z}{r^{2}}\right)$, compare [2, Theorem 2.9, p. 2580]. This leads to estimates for small $r$, equivalently for large $\hat{r}$, such as

$$
|D \chi|_{\delta}=\frac{1}{r^{2}}|\hat{D} \chi|_{\hat{\delta}} \lesssim \frac{1}{r^{2}} \hat{\rho} \hat{r}^{-\gamma-1}=\rho r^{\gamma-3}, r \rightarrow 0 .
$$

From this and the known asymptotic behaviour of extreme Kerr-Newman one obtains, when the decay exponent $\lambda$ of $K$ (see (2.1)) satisfies $\lambda \geq 2 \gamma+1,{ }^{4}$

$$
v-\tilde{v}=O\left(r^{2 \gamma-2}\right) .
$$

This allows us to estimate the contribution of the term $D \varphi_{\eta}(v-\tilde{v})$ of $(2.19)$ in the subregion $\rho \geq z$ of the integral (2.21) as

$$
\int_{\eta / 2}^{\eta} \frac{r^{4}}{\rho^{4} r^{2}}\left(r^{2 \gamma-2}\right)^{2} r^{2} d r=O\left(\eta^{4 \gamma-3}\right) \rightarrow_{\eta \rightarrow 0} 0 \text { provided that } \gamma>3 / 4
$$

This explains our ranges of $\lambda$ and $\gamma$ in (2.1) and (2.3).

A detailed presentation, with some simplifications of the argument of [3], will be given elsewhere $[4,5]$.

Acknowledgements: We are grateful to S. Dain and E. Delay for many useful discussions, and for sharing their calculations of the potentials for the extreme Kerr-Newman solution.

\section{References}

[1] D. Brill, On the positive definite mass of the Bondi-Weber-Wheeler timesymmetric gravitational waves, Ann. Phys. 7 (1959), 466-483.

\footnotetext{
${ }^{4}$ For $\lambda \leq 2 \gamma+1$ the dominating behaviour in (2.12) is governed by $\lambda$, which leads to $v-\tilde{v}=O\left(r^{\lambda-3}\right)$ and the necessity to impose $\lambda>5 / 2$, as in vacuum [3, p. 2602].
} 
[2] P.T. Chruściel, Mass and angular-momentum inequalities for axisymmetric initial data sets. I. Positivity of mass, Annals Phys. 323 (2008), 2566-2590, doi:10.1016/j.aop.2007.12.010, arXiv:0710.3680 [grqc].

[3] P.T. Chruściel, Y. Li, and G. Weinstein, Mass and angular-momentum inequalities for axi-symmetric initial data sets. II. Angular momentum, Annals Phys. 323 (2008), 2591-2613, doi:10.1016/j.aop.2007.12.011, arXiv:0712.4064v2 [gr-qc].

[4] J. Lopes Costa, A Dain inequality with charge, in preparation.

[5] — On black hole uniqueness theorems, Ph.D. thesis, Oxford, in preparation.

[6] S. Dain, Proof of the angular momentum-mass inequality for axisymmetric black holes, Jour. Diff. Geom. 79 (2006), 33-67, arXiv:gr-qc/0606105. MR MR2414749

[7] _ The inequality between mass and angular momentum for axially symmetric black holes, Int. Jour. Modern Phys. D 17 (2008), 519-523. MR MR2441278 (2009e:83085)

[8] G.W. Gibbons and G. Holzegel, The positive mass and isoperimetric inequalities for axisymmetric black holes in four and five dimensions, Class. Quantum Grav. 23 (2006), 6459-6478, arXiv:gr-qc/0606116. MR MR2272015

[9] J. Hennig, C. Cederbaum, and M. Ansorg, A universal inequality for axisymmetric and stationary black holes with surrounding matter in the Einstein-Maxwell theory, (2008), arXiv:0812.2811 [gr-qc].

[10] G. Weinstein, $N$-black hole stationary and axially symmetric solutions of the Einstein/Maxwell equations, Commun. Part. Diff. Eqs. 21 (1996), 1389-1430.

[11] G. Weinstein and S. Yamada, On a Penrose inequality with charge, arXiv:math.dg/0405602. 